\title{
Peritoneal Inclusion Cysts after Gunshot Wound William Weber
}

\author{
Section of Emergency Medicine, The University of Chicago, United States. \\ weberwilliamd@gmail.com
}

*Corresponding Author: William Weber, 5841 S. Maryland Ave, MC 5068, Chicago, Illinois, 60637, United States.

\section{Abstract}

Female patients with a history of abdominal trauma or surgery can present with abdominal pain and swelling secondary to peritoneal inclusion cysts. These cysts develop as a result of faulty absorption of physiologic ovarian secretions by the peritoneum after irritation from trauma. Cyst size can be decreased by hormonal pharmacotherapy, but cysts are often difficult to eradicate, even with surgical management.

\section{Clinical Image}

A 32-year-old woman with a history of gunshot wound to the abdomen and exploratory laparotomy six months prior presented to the emergency department with a four day history of worsening, sharp abdominal pain and abdominal swelling. She denied fevers, emesis, diarrhea, dysuria, and vaginal bleeding. She had not been sexually active since the surgery and her last menstrual period ended one week prior.

On exam, the woman had a tender protuberance of her right lower quadrant without rebound. She denied a past history of this mass. No herniation was present on palpation of her abdominal wall with Valsalva maneuver. Urine pregnancy test was negative. CT scan showed multiple, cystic fluid collections, with the largest one measuring $9 \mathrm{~cm}$ by $7 \mathrm{~cm}$ by $14 \mathrm{~cm}$ and displacing both bowel and ovary.

Reactive mesothelial cells in the peritoneal cavity can encapsulate the free fluid, forming a cyst. The fluid collections were diagnosed as peritoneal inclusion cysts, fluid filled masses that can form after peritoneal irritation. Ovaries secrete physiologic fluid during ovulation that is typically absorbed by the peritoneum. During inflammatory states (e.g., after exploratory laparotomy), the peritoneum can decrease in its absorptive ability. The fluid can become trapped and encapsulated within a cyst lined with reactive mesothelial cells. During ovulation, cysts can grow rapidly due to increased fluid secretion by the ovaries. Patients usually present with pain or abdominal fullness, but can also present with a palpable mass. Diagnosis is usually made by CT scan ${ }^{1}$. While peritoneal inclusion cysts have no malignant potential, they can cause substantial pain and infertility ${ }^{2}$.

Treatment can include hormonal therapies to decrease fluid production as well as mechanical cyst drainage. Surgical removal of the cysts is a more definitive

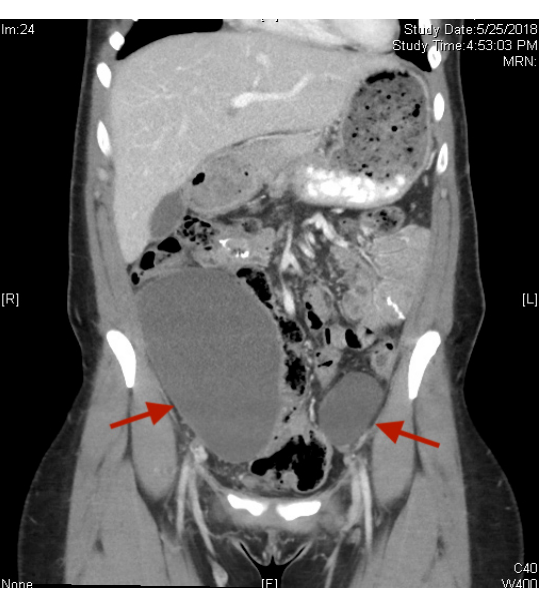

Figure 1. Computed tomography of abdomen seven weeks prior to presentation with no evidence of cysts. Figure 2. Computed tomography of abdomen showing multiple peritoneal inclusion cysts in the pelvic cavity (arrows). 
Peritoneal Inclusion Cysts after Gunshot Wound

treatment, but around $50 \%$ of women will still have recurrence ${ }^{3}$.

\section{REFERENCES}

[1] Jain KA. Imaging of peritoneal inclusion cysts. American Journal of Roentgenology. 2000 Jun;174(6):1559-63.
[2] Vallerie AM, Lerner JP, Wright JD, Baxi LV. Peritoneal inclusion cysts: a review. Obstetrical \& gynecological survey. 2009 May 1;64(5):321-34.

[3] Jones SA, Salicco JM, Byers MS. Pelvic pain and history of previous pelvic surgery. Proceedings (Baylor University. Medical Center). 2003 Jan;16(1):121.

Citation: William Weber. Peritoneal Inclusion Cysts after Gunshot Wound. Archives of Emergency Medicine and Intensive Care. 2018; 1(1): 27-28.

Copyright: (C) 2018 William Weber. This is an open access article distributed under the Creative Commons Attribution License, which permits unrestricted use, distribution, and reproduction in any medium, provided the original work is properly cited. 